\title{
CARACTERÍSTICAS E DIMENSÕES DO VOLUME DE UM SOLO MOLHADO SOB GOTEJAMENTO SUPERFICIAL E SUBSUPERFICIAL ${ }^{1}$
}

\author{
Carlos César Pereira Nogueira ${ }^{2}$, Eugênio Ferreira Coelho ${ }^{3} \&$ Moisés Custódio Saraiva Leão ${ }^{4}$
}

\begin{abstract}
RESUMO
Objetivou-se, com este trabalho, estabelecer comparações entre os bulbos molhados por gotejadores localizados na superfície do solo, e enterrados no final do ciclo da irrigação, além de avaliar modelos matemáticos para estimativa das dimensões desses volumes molhados. O estudo foi conduzido em um solo Podzólico Vermelho-Amarelo, em Fortaleza, CE. Em ambos os casos, foi aplicado simultaneamente, o volume de $4,33 \times 10^{-3} \mathrm{~m}^{3}(4,33 \mathrm{~L})$ de água numa vazão de $7,22 \times 10^{-7} \mathrm{~m}^{3} \mathrm{~s}^{-1}$ $\left(2,6 \mathrm{~L} \mathrm{~h}^{-1}\right)$. O monitoramento da dinâmica da água no solo foi realizado através de tensiômetros. Ao final de dois ciclos de irrigação observou-se, no volume molhado sob gotejamento enterrado, um teor de água disponível variando entre 44 a $29 \%$ maior que no caso do emissor superficial. Dentre os modelos testados o de Schwartzmass \& Zur apresentou melhor correlação com as determinações experimentais.
\end{abstract}

Palavras-chave: subirrigação, bulbo molhado, modelos

\section{CHARACTERISTICS AND DIMENSIONS OF THE VOLUME OF WETTED SOIL UNDER SURFACE AND SUBSURFACE DRIP IRRIGATION}

\begin{abstract}
The objective of this work was to establish comparisons among characteristics and dimensions of wetted volumes under surface and subsurface drippers at the end of an irrigation cycle and to evaluate mathematical models for predicting dimensions of these wetted volumes. The study was carried out in a Yellow-Red Podzol in Fortaleza-CE. Water volume of $4.3 \times 10^{-3} \mathrm{~m}^{3}(4.33 \mathrm{~L})$ was applied by drippers with flow rate of $7.22 \times 10^{-7} \mathrm{~m}^{3} \mathrm{~s}^{-1}\left(2.6 \mathrm{~L} \mathrm{~h}^{-1}\right)$ during the studied irrigation cycles. Soil water dynamics monitoring was accomplished by tensiometers. Measurements of soil-water potential were done before the irrigation event and during two irrigation cycles. At the end of the two irrigation cycles the water content that was available in the wetted volume under the buried dripper was 44 to $29 \%$ larger than the water content that was available in the wetted volume under the surface dripper. The Schwartzmass \& Zur model resulted in a better approximation to the experimental data.
\end{abstract}

Key words: Sub irrigation, wet bulb, models

\footnotetext{
Recebido em 10/02/2000, Protocolo 017/00

${ }^{1}$ Extraído da Dissertação apresentada ao DENA/UFC, pelo primeiro autor, para obtenção do título de Mestre em Agronomia (Irrigação e Drenagem)

${ }^{2}$ M.S. em Agronomia (Irrigação e Drenagem) do DENA/ UFC, Av. Capitão Claro 142, CEP 64200 - 500, Parnaíba, PI. Fone: (0xx86) 321 1406. E-mail:nogueira@cpamn.embrapa.br

${ }^{3}$ Ph.D. em Irrigação e Drenagem, Pesquisador da EMBRAPA/CNPMF, CEP 44380 - 000, Cruz das Almas, BA. Fone: (0xx75) 7212120. E-mail ecoelho@cnpmf.embrapa.br

${ }^{4}$ Ph.D. em Irrigação e Drenagem, Professor da UFC, Departamento de Engenharia Agrícola, Bloco 804, CEP 60455 - 760 , Fortaleza, CE. Fone: (0xx85) 2889756
} 


\section{INTRODUÇÃO}

A demanda crescente por recursos de água acentua a necessidade de seu manejo cada vez mais racional, de modo a assegurar a integridade, a produtividade, a diversidade e a vitalidade dos sistemas aquáticos e de suas bacias (Bucks, 1995). A irrigação por gotejamento oferece oportunidades para $\mathrm{o}$ atendimento dessas necessidades, proporcionando melhoria na qualidade da colheita, maior rendimento e eficiência no uso da água; além disso permite, ainda, a aplicação simultânea de fertilizantes possibilitando, assim, a utilização mais racional desses fertilizantes, quando comparada com as outras formas de aplicação.

A irrigação por gotejamento é caracterizada pela aplicação de pequenas quantidades de água em alta freqüência e diretamente na zona radicular, mantendo a umidade próximo ao limite superior de disponibilidade de água, em um volume de solo (Nir, 1982; Dasberg \& Bresler, 1985). Este sistema pode ser classificado, segundo a posição de instalação da linha de emissores, em superficial, quando os emissores se encontram na superfície do solo, e subsuperficial, quando os emissores se localizam abaixo da superfície.

Os sistemas de irrigação por gotejamento superficial constituem o método de aplicação mais utilizado entre os sistemas por gotejamento. As vantagens do gotejamento superficial incluem a facilidade de instalação, inspeção, mudança de posição e limpeza de emissores, além da possibilidade de verificação dos padrões de umidade na superfície do solo e da medida da vazão dos emissores, individualmente.

O sistema de gotejamento subsuperficial já foi criticado por Goldberg et al. (1986); entretanto, com o desenvolvimento de novas tecnologias que garantem a uniformidade de aplicação da água próximo a $100 \%$, as razões dessa crítica foram minimizadas. Este sistema se caracteriza pelo fato da emissão de água ser feita subsuperficialmente, com a frente de umidade tangenciando a superfície do solo (dependendo da profundidade), evitando a exposição de superfície molhada e reduzindo, ainda mais, as perdas por evaporação.

A distribuição de água no bulbo molhado é dependente do volume total de água aplicado, da vazão, da posição do gotejador (na superfície do solo ou enterrado), da disposição dos gotejadores (fonte pontual ou fonte em linha), das condições iniciais e de contorno, das propriedades físicas do solo e de sua distribuição espacial, da atividade do sistema radicular das plantas e do manejo da irrigação (Brandt et al., 1971; Levin \& van Rooyen, 1979; Taghavi et al., 1984; Dasberg \& Bresler, 1985; Goldberg et al., 1986; Coelho \& Or, 1996).

As informações sobre a distribuição da água no bulbo molhado são de grande importância, tanto para o dimensionamento dos sistemas de irrigação como no seu manejo, visto que a determinação do espaçamento entre emissores, a localização de sensores de umidade, a definição das zonas de diferentes intensidades de absorção de água e nutrientes, requerem o conhecimento da dinâmica de água no bulbo molhado.

Ben-Asher et al. (1986) propuseram um modelo com base analítica, que assume que o bulbo molhado formado por um gotejador posicionado na superfície do solo tem a forma aproximada de uma semi-esfera; o raio molhado deste bulbo é calculado em função da vazão do gotejador e da variação do teor de água antes da irrigação, e o maior teor de água após a irrigação.
Ben-Asher \& Phene (1993) baseados no modelo semiesférico proposto por Ben-Asher et al. (1986) propuseram um modelo esférico para o bulbo, formado por um gotejador em posição subsuperficial.

Para Schwartzmass \& Zur (1985) o volume de solo molhado depende da condutividade hidráulica do solo, da vazão do emissor e do total de água aplicado no solo. Keller \& Bliesner (1990) citam o uso de modelos matemáticos, tais como o de Schwartzmass $\&$ Zur (1985) mas sugerem cautela no uso dos mesmos, dada a variabilidade espacial das propriedades físicas do solo.

O objetivo deste trabalho foi estabelecer comparações entre os volumes de água remanescentes, dentro dos limites de água disponível à cultura, nos dois sistemas de irrigação ao final de dois ciclos de irrigação e, também, testar a adaptabilidade de modelos para o dimensionamento do bulbo molhado.

\section{MATERIAL E MÉTODOS}

O experimento foi instalado na área física do Laboratório de Hidráulica do Departamento de Engenharia Agrícola da Universidade Federal do Ceará, em Fortaleza, CE, em solo Podzólico Vermelho-Amarelo. O sistema constou de duas linhas de gotejadores do tipo "in line", ambas com as mesmas dimensões: comprimento de $20 \mathrm{~m}$ e diâmetro interno de $0,0156 \mathrm{~m}$. Foi aplicado, simultaneamente para cada caso, o volume 4,33 x $10^{-3} \mathrm{~m}^{3}(4,33 \mathrm{~L})$ de água com gotejadores de vazão de $7,22 \times 10^{-7} \mathrm{~m}^{3} \mathrm{~s}^{-1}\left(2,6 \mathrm{~L} \mathrm{~h}^{-1}\right)$, espaçados 1,6 $\mathrm{m}$. A primeira linha foi enterrada a $0,2 \mathrm{~m}$ de profundidade e a outra deixada na superfície do solo. Para realização deste trabalho, foi necessária a determinação de algumas características físico-hídricas do solo, conforme Tabela 1.

Tabela 1. Parâmetros físicos do solo levantados durante os meses de outubro e novembro de 1997

\begin{tabular}{|c|c|c|c|c|}
\hline \multirow{2}{*}{$\begin{array}{l}\text { Profundidade } \\
\text { (m) }\end{array}$} & \multirow{2}{*}{$\begin{array}{c}\text { Densidade } \\
\text { do Solo } \\
\left(\mathrm{kg} \mathrm{dm}^{-3}\right)\end{array}$} & \multicolumn{2}{|c|}{ Umidade do Solo } & \multirow{2}{*}{$\begin{array}{l}\text { Umidade de } \\
\text { Saturaçãa } \\
\left(\mathrm{cm}^{3} \mathrm{~cm}^{-3}\right)\end{array}$} \\
\hline & & $10 \mathrm{kPa}^{*}$ & $1500 \mathrm{kPa}^{*}$ & \\
\hline 0,0 a 0,1 & 1,50 & 0.19 & 0,078 & 0,39 \\
\hline 0,1 a 0,2 & 1,58 & 0,18 & 0,082 & 0,30 \\
\hline 0,2 a 0,3 & 1,56 & 0,19 & 0,088 & 0,31 \\
\hline 0,3 a 0,4 & 1,46 & 0,18 & 0,085 & 0,31 \\
\hline 0,4 a 0,5 & 1,44 & 0,19 & 0,084 & 0,31 \\
\hline 0,5 a 0,6 & 1,35 & 0,18 & 0,085 & 0,31 \\
\hline 0,6 a 0,7 & 1,34 & 0,23 & 0,130 & 0,39 \\
\hline 0,7 a 0,8 & 1,44 & 0,29 & 0,170 & 0,52 \\
\hline 0,8 a 0,9 & 1,50 & 0,29 & 0,180 & 0,49 \\
\hline 0,9 a 1,0 & 1,51 & 0,28 & 0,190 & 0,44 \\
\hline
\end{tabular}

* Valores obtidos da curva de retenção

A condutividade hidráulica saturada $\left(\mathrm{k}_{\mathrm{S}}\right)$ foi determinada nas profundidades de 0,2,0,4 e $0,6 \mathrm{~m}$, utilizando-se amostras indeformadas, três por camada, pelo método do permeâmetro de carga constante, segundo Libardi (1995). Calculou-se uma média harmônica dos valores, segundo a expressão:

$$
\mathrm{k}_{\mathrm{s}}=\frac{\mathrm{L}_{1}+\mathrm{L}_{2}+\cdots \mathrm{L}_{\mathrm{n}}}{\frac{\mathrm{L}_{1}}{\mathrm{k}_{1}}+\frac{\mathrm{L}_{2}}{\mathrm{k}_{2}}+\cdots+\frac{\mathrm{L}_{\mathrm{n}}}{\mathrm{k}_{\mathrm{n}}}}
$$

em que L é a espessura da camada de condutividade hidráulica saturada $\mathrm{k}_{\mathrm{n}}$. 
Para monitoramento da dinâmica da água no solo foi instalada, em cada uma das linhas de gotejadores, uma bateria com 16 tensiômetros, em uma malha que teve início a $5 \mathrm{~cm}$ do gotejador e foi até $35 \mathrm{~cm}$ de distância do ponto de emissão na horizontal, até a profundidade de $90 \mathrm{~cm}$ na vertical. Os tensiômetros foram distribuídos ao longo de três planos cortantes ao bulbo molhado, passando pelo ponto de emissão, de maneira que a projeção desses planos em um único plano produziu a Figura 1.

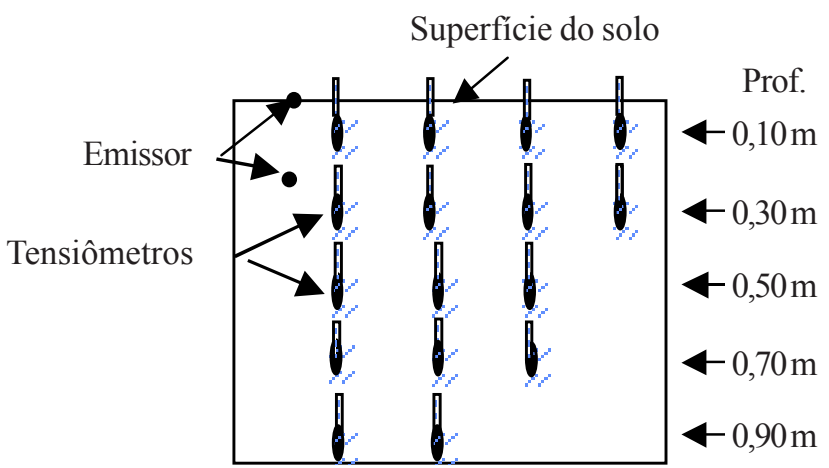

Figura 1. Projeção em um só plano, dos três planos de distribuição de tensiômetros no volume molhado

A dinâmica da água no solo na condição de fluxo não permanente foi analisada durante dois ciclos de irrigação, obedecendo ao seguinte intervalo de leitura de tensiômetros: $5 \mathrm{~h}$ após a primeira irrigação; imediatamente antes da segunda irrigação, o que corresponde a $48 \mathrm{~h}$ após a primeira irrigação e $48 \mathrm{~h}$ após a segunda irrigação. Devido ao baixo teor de água do solo no estado natural antes da primeira irrigação, impossibilitando o funcionamento dos tensiômetros, optou-se pelo método da estufa, para determinação da umidade do solo, observando-se que toda a região em estudo se encontrava com a umidade próximo ao ponto de murcha permanente.

A equação proposta por Ben-Asher et al. (1986) para o dimensionamento do bulbo molhado formado por um gotejador posicionado na superfície, é dada por:

$$
\mathrm{R}_{\mathrm{DI}}=\frac{\left(\frac{3 \mathrm{Q}}{2 \pi}\right)^{1 / 3}}{\Delta \theta_{\mathrm{DI}}}
$$

sendo que: $\mathrm{R}_{\mathrm{DI}}$ é o raio molhado na superfície semi-esférica irrigada (m); Q é o total de água aplicado $\left(\mathrm{m}^{3}\right)$ e $\Delta \theta_{\mathrm{DI}}$ é a variação entre o teor de água antes da irrigação e o teor de água no momento da coleta da informação.

A equação proposta por Ben-Asher \& Phene (1993) para dimensionamento do bulbo formado pelo emissor enterrado, é dada por:

$$
\mathrm{R}_{\mathrm{SDI}}=\frac{\left(\frac{3 \mathrm{Q}}{4 \pi}\right)^{1 / 3}}{\Delta \theta_{\mathrm{SDI}}}
$$

em que: $\mathrm{R}_{\mathrm{SDI}}$ é o raio molhado na superfície semi-esférica irrigada (m); Q é o total de água aplicado $\left(\mathrm{m}^{3}\right)$; e $\Delta \theta_{\mathrm{SDI}}$ refere-se à diferença do teor de água antes da irrigação e o teor de água no momento da análise.

O modelo proposto por Schwartzmass \& Zur (1985) não faz distinção quanto à posição do emissor; portanto, o diâmetro do bulbo molhado na superfície é o mesmo para o diâmetro medido a $0,2 \mathrm{~m}$ de profundidade, como é o caso do emissor enterrado. Sua equação para o dimensionamento do diâmetro do bulbo molhado é dada por:

$$
\mathrm{z}^{\prime}=\mathrm{K}_{1}\left(\mathrm{~V}_{\mathrm{w}}\right)^{0,63}\left(\mathrm{k}_{\mathrm{s}} / \mathrm{q}\right)^{0,45}
$$

$\mathrm{e}$

$$
\mathrm{w}^{\prime}=\mathrm{K}_{2}\left(\mathrm{~V}_{\mathrm{w}}\right)^{0,22}\left(\mathrm{k}_{\mathrm{s}}\right)^{-0,17}
$$

Da relação entre $w^{\prime}$ e $z$, obtém-se:

$$
\mathrm{W}=\mathrm{K}_{3}\left(\mathrm{z}^{\prime}\right)^{0,35}(\mathrm{q})^{0,33}\left(\mathrm{k}_{\mathrm{s}}\right)^{-0,33}
$$

em que:

$$
\begin{array}{ll}
\mathrm{w} & \text { - coeficiente empírico } \\
\mathrm{z} & \text { - distância vertical para frente de molhamento }(\mathrm{m}) \\
\mathrm{W} & \text { - diâmetro do bulbo molhado }(\mathrm{m}) \\
\mathrm{K}_{1} & \text { - coeficiente empírico }=29,2 \text { para unidades métricas } \\
\mathrm{V}_{\mathrm{w}} & \text { - volume de água aplicado }(\mathrm{L}) \\
\mathrm{k}_{\mathrm{s}} & \text { - condutividade hidráulica saturada }\left(\mathrm{m} \mathrm{s}^{-1}\right) \\
\mathrm{q} & \text { - vazão do emissor pontual }\left(\mathrm{L} \mathrm{h}^{-1}\right) \\
\mathrm{K}_{2} & \text { - coeficiente empírico }=0,031 \text { para unidades métricas } \\
\mathrm{K}_{3} & \text { - coeficiente empírico }=0,0094 \text { para unidades métricas. }
\end{array}
$$

Para efeito de cálculo, consideraram-se as isolinhas de - $40 \mathrm{kPa}$ (400 cm coluna de água), o que equivale aproximadamente a 50\% da água disponível às culturas, como limite dos bulbos molhados. Foram consideradas as tensões tomadas $5 \mathrm{~h}$ após a irrigação, por ser o tempo limite da expansão do bulbo molhado determinado no experimento.

\section{RESULTADOS E DISCUSSÃO}

Compararam-se os volumes de água dentro das isolinhas de $-40 \mathrm{kPa}$, encontrados nas seções centrais dos bulbos (fatia cortada no centro do bulbo em posição vertical com $1,0 \mathrm{~cm}$ de espessura) tomadas $48 \mathrm{~h}$ após a primeira irrigação, apresentadas nas Figuras 2A (emissor superficial) e B (emissor subsuperficial) e $48 \mathrm{~h}$ após a segunda irrigação, apresentadas nas Figuras 3A (emissor superficial) e B (emissor subsuperficial). Obteve-se, para o caso do emissor superficial no final do primeiro ciclo, o volume de $0,312 \times 10^{-3} \mathrm{~m}^{3}$, enquanto para o emissor subsuperficial o volume encontrado foi de $0,450 \times 10^{-3} \mathrm{~m}^{3}$. No final do segundo ciclo, os volumes encontrados foram de 0,446 $\mathrm{x} 10^{-3} \mathrm{~m}^{3}$ para o emissor superficial e de $0,576 \times 10^{-3} \mathrm{~m}^{3}$ para o emissor subsuperficial. O teor de água remanescente no bulbo formado pelo emissor subsuperficial, no final do segundo ciclo, foi $29 \%$ superior ao encontrado no bulbo formado pelo emissor superficial.

Considerando-se o menor volume disponível para distribuição de água no caso do gotejamento superficial, poderia esperar-se maior umidade média nesse volume, comparado com o gotejamento subsuperficial (Ben-Asher et al., 1986). Entretanto, 
(A)

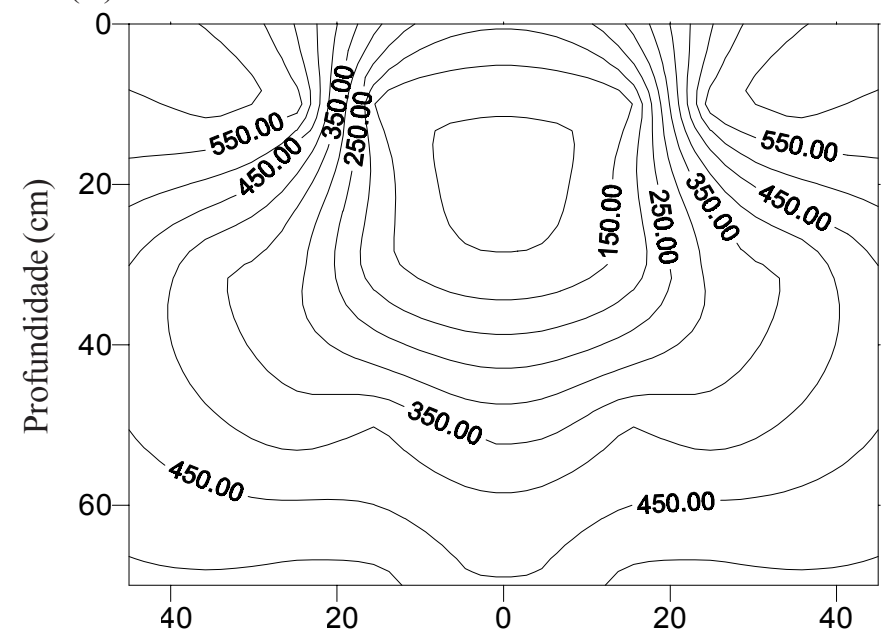

(B)

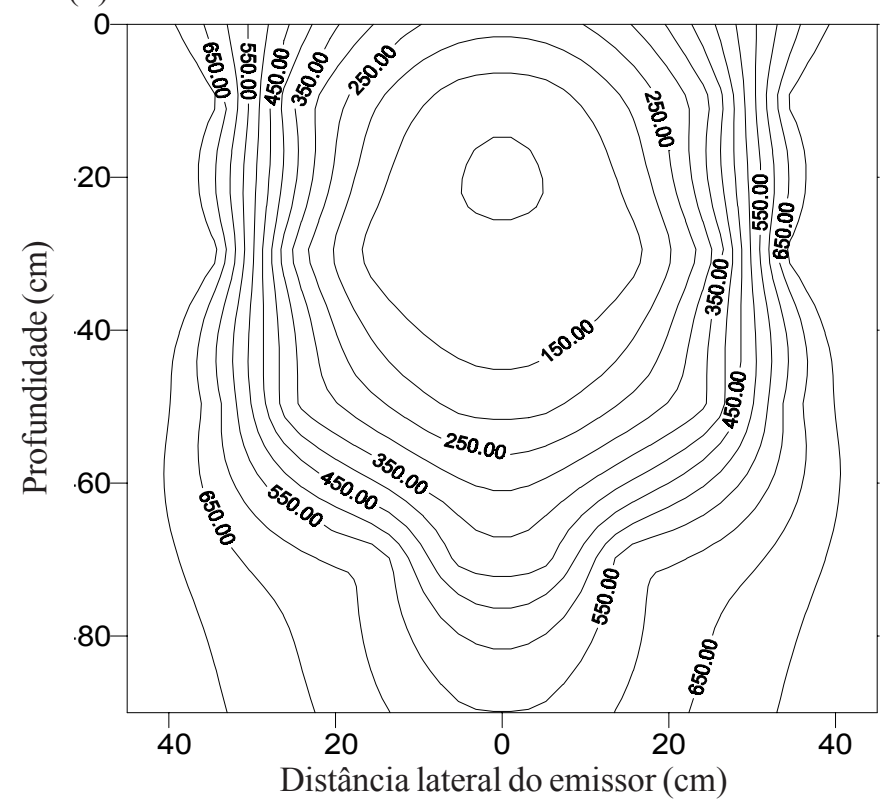

Figura 2. Perfil de distribuição de tensão de umidade (cm c a), $48 \mathrm{~h}$ após a primeira irrigação A. Emissor superficial, B. Emissor subsuperficial

na região do bulbo delimitada pelas isolinhas de $-40 \mathrm{kPa}$ à umidade média foi maior para o gotejamento subsuperficial, fato que se deve à maior resistência à infiltração, no caso do gotejador subsuperficial mantendo, assim, maiores teores de água próximo ao gotejador. Deve-se acrescentar, também, a ocorrência de maior evaporação no sistema superficial comparado ao sistema subsuperficial e a variação da textura do solo ao longo do perfil. A maior parte do bulbo formado pelo emissor superficial abrangeu uma areia franca, enquanto no segundo caso o emissor se localiza na região de transição do perfil, sendo o hemisfério inferior do bulbo formado por solo franco-arenoso com maior capacidade de armazenamento. Resultados semelhantes foram obtidos por Phene (1989) apud Ben-Asher \& Phene (1993) e Phene (1995), cujo comportamento indica a possibilidade de maior concentração do sistema radicular, ou seja, maior densidade de comprimento de raízes no volume estudado para o gotejamento subsuperficial em relação ao bulbo molhado, referente ao gotejamento subsuperficial.
(A)

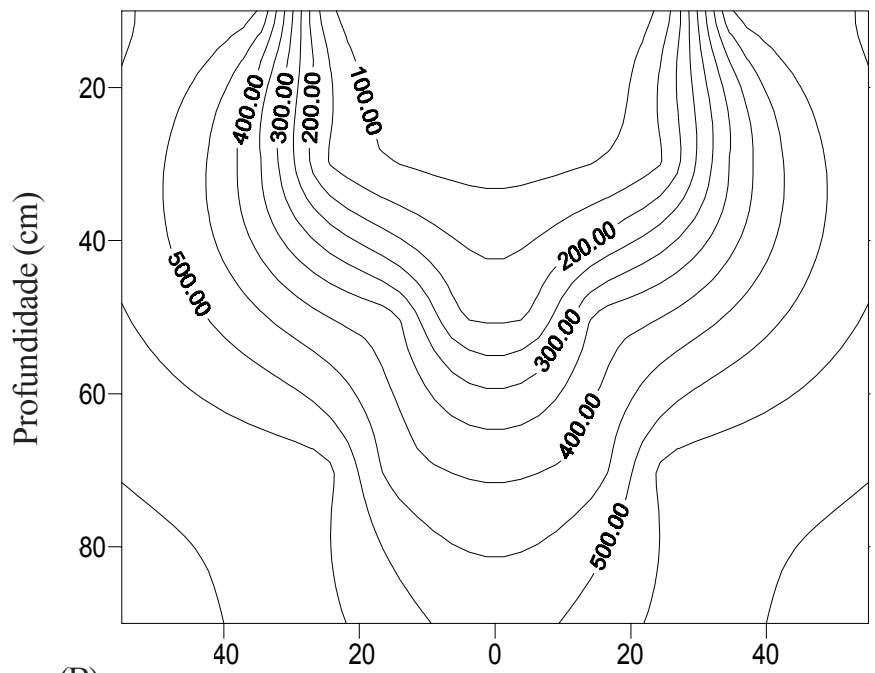

(B)

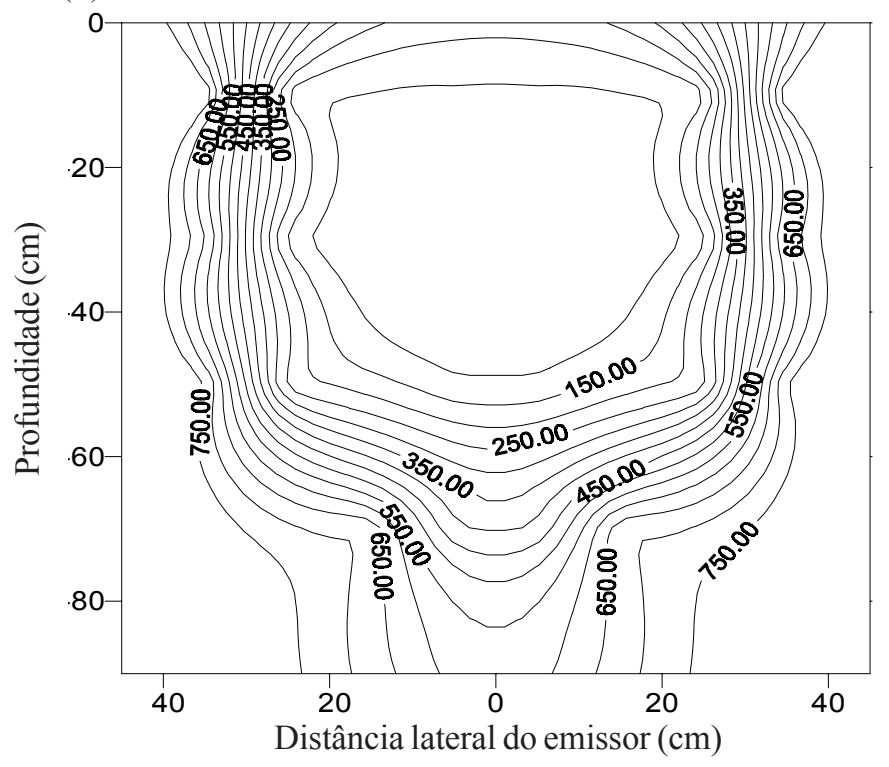

Figura 3. Perfil de distribuição de tensão de umidade (cm c a), $48 \mathrm{~h}$ após a segunda irrigação A. Emissor superficial, B. Emissor subsuperficial

As Figuras de 2 e 3 mostram pequena diferença na distribuição horizontal de água no bulbo molhado entre $48 \mathrm{~h}$ após a primeira, e $48 \mathrm{~h}$ após a segunda irrigação, para ambos os gotejadores superficiais e subsuperficiais, o que se acentua na direção vertical, por onde, pelas isolinhas de $-40 \mathrm{kPa}$ chegam a 0,7 $\mathrm{m}$ para os dois casos, indicando a possibilidade de perdas por percolação, quando se considera que grande parte das culturas tem seus sistemas radiculares efetivos a profundidades de 0,6 m. Em ambos os sistemas de gotejamento, houve uma expansão significativa do volume de solo contornado pelas isolinhas de teores de água em consideração. Verificou-se, também, maior expansão do volume de solo dentro das isolinhas de $-10 \mathrm{kPa}$ (limite superior da disponibilidade de água do solo) em torno do gotejador subsuperficial, indicando um volume maior do bulbo, com condições mais adequadas à absorção de água pelas raízes, quando comparado ao bulbo molhado sob gotejamento superficial. 
Da aplicação do modelo de Schwartzmass \& Zur (1985) o valor para o diâmetro molhado foi de $0,49 \mathrm{~m}$ (esta informação se aplica aos dois casos, já que o autor não faz considerações quanto à posição do emissor). Com o modelo de Ben-Asher et al. (1986), obteve-se 1,26 m como valor do diâmetro do bulbo para com emissor na superfície, e 1,00 m para o caso do emissor enterrado a $0,2 \mathrm{~m}$ de profundidade.

Observa-se, através das Figuras 4A e B, o perfil de distribuição de tensão de umidade com base nos dados colhidos $5 \mathrm{~h}$ após a irrigação. Os diâmetros dos bulbos molhados medidos na horizontal, a $0,20 \mathrm{~m}$ de profundidade, assumindo $\theta=13 \%$ como limite da frente de molhamento, equivalente à tensão de $-40 \mathrm{kPa}$, foram de $0,60 \mathrm{~m}$ para o emissor na superfície e de $0,57 \mathrm{~m}$ para o emissor enterrado. O modelo de Schwartsmass $\&$ Zur (1985) estimou um valor $20 \%$ inferior ao obtido experimentalmente no caso do emissor superficial. No caso do emissor enterrado, esse valor foi $14 \%$ menor que o resultado experimental.

O modelo de Ben-Asher et al. (1986) para o caso do emissor superficial, estimou um diâmetro de $1,26 \mathrm{~m}$, superestimando em $110 \%$ e, para o emissor enterrado, o modelo estimou um valor $75 \%$ maior que o resultado experimental. O modelo de Schwartzmass \& Zur (1985) tem, como variável, a condutividade

\section{(A)}

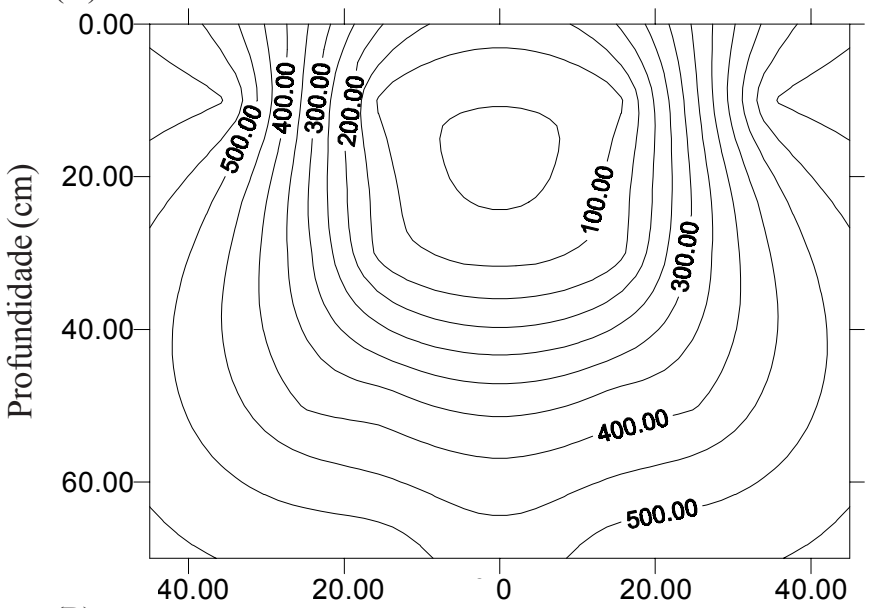

(B)

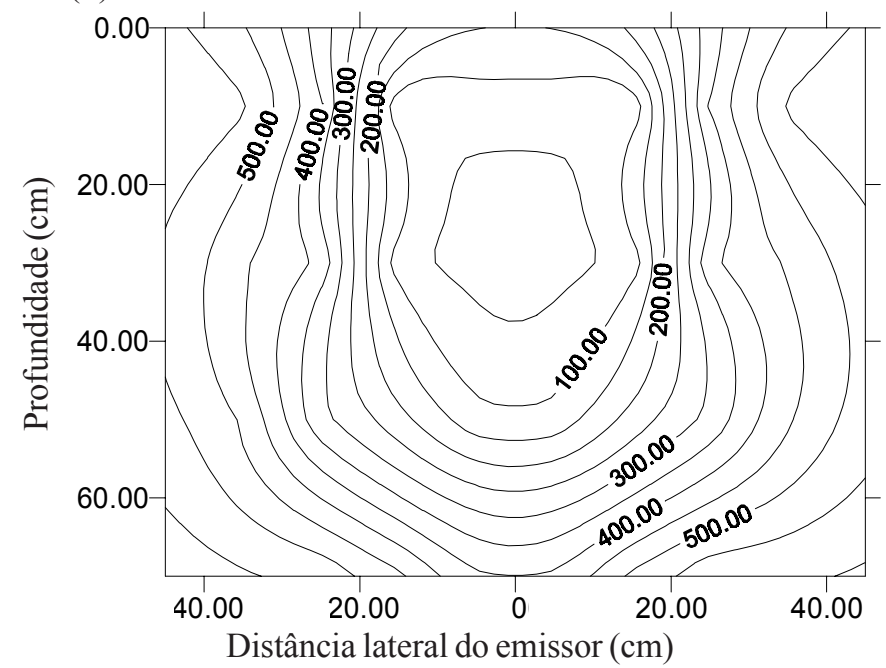

Figura 4 . Perfil de distribuição de tensão de umidade ( $\mathrm{cm} \mathrm{c} \mathrm{a)}$ A. Emissor superficial, B. Emissor subsuperficial, 5 h após a primeira irrigação hidráulica saturada do solo; portanto, sensível ao efeito da compactação, e pode variar em função do método usado para determinação. A condutividade hidráulica saturada que os autores usam representa todo o perfil onde se insere o volume molhado, não levando em conta a variabilidade espacial do solo, enquanto o modelo de Ben-Asher et al. (1986), para gerar a fórmula, assumiu que o teor de água do solo é constante dentro do bulbo molhado. Na prática, o teor de água do solo varia radialmente. Deve-se considerar, também, que o modelo de Ben-Asher et al. (1986) não utiliza nenhuma variável de solo. Esse fato contribui para explicar sua grande imprecisão, pois se presume que os autores tenham tomado, por base, experimentos realizados em solo sob condições naturais, isto é, sem os problemas de adensamento ocorridos no caso desse estudo, como pode ser visto na Tabela 1.

\section{CONCLUSÕES}

1. Para as condições experimentais desse estudo, a localização dos emissores abaixo da superfície mostrou-se mais eficiente que a localização superficial, no que tange à reserva de umidade disponível no solo.

2. Dentre os modelos testados, o de Schwartsmass \& Zur (1985) apresentou resultados aproximados às determinações experimentais podendo, portanto, ser utilizado nas condições experimentais, enquanto o modelo de Ben-Asher et al. (1986) não se mostrou satisfatório.

\section{REFERÊNCIAS BIBLIOGRÁFICAS}

BEN-ASHER, J.; CHARACH, C.; ZEMEL, A. Infiltration and water extractiction from trikle irrigation source: The effective hemisphere model. Soil Science Society America Journal, Madison, v.50, n.4. p.882-887, 1986.

BEN-ASHER, J.; PHENE, C.J. The effect of surface drip irrigation on soil water regime evaporation and transpiration. In: INTERNATIONAL CONFERENCE ONIRRIGATION, 6, 1993, Tel-Aviv, Israel, Proceedings... Tel-Aviv: 1993, p.35-42.

BRANDT, A.; BRESLER, E.; DINER, N., BEN-ASHER, I.; HELLER, J.; GOLDBERGM, D. Infiltration from a trickle source: I. Mathematical models. Soil Science Society American Proceedings, Madison, v.35, p.683-689, 1971.

BUCKS, D.A. Historical devlopment in microirrigation. In: INTERNATIONAL MICROIRRIGATION CONGRESS, 5.,1995, Orlando, Florida, Proceedings... St.Joseph: 1995. p.1-5.

COELHO, E.F.; OR, D. Applicability of analytical solutions for flow from point sources to drip irrigation management. Soil Science Society of America Journal, Madison, v.61, n.4, p.1331-1341, 1996.

DASBERG, S.; BRESLER, E. Drip irrigation manual. Logan: International Irrigation Center, 1985, 95p.

GOLDBERG, D.; GORNAT, B.; RIMOM, D. Drip irrigation: Principles, design and agricultural practices. Israel: DIS publications, 1986.

KELLER, J.; BLIESNER, D.R. Sprinkler and trickle irrigation. New York: von Nostrand Reinhold, 1990. 652p.

LEVIN, I.; van ROOYEN, F.C. The effect of discharge rate and intermittent water application by point-source irrigation on the soil moisture distribution pattern. Soil Science Society of America Journal, Madison, v.43, p.8-16, 1979. 
LIBARDI, P.L. Dinâmica da água no solo. Piracicaba: (s.n). 1993, 497p.

NIR, D. Drip irrigation. In: FINKEL, H.J. CRC Handbook of irrigation tecnology. Boca Raton: CRC Press, 1982. v.1, p.247-298.

PHENE, C. J. The sustainability and potential of subsurface drip irrigation. In: PROCEEDINGS OF THE INTERNATIONAL MICROIRRIGATION CONGRESS, 5. Orlando, Florida. April 2-6, 1995. p.359-367.
SCHWARTZMASS. M.; ZUR, B. Emitter spacing and geometry of wetted soil volume. Journal of Irrigation and Drainage Engineering. New York: ASCE 112. v.3, p.242-253, 1985.

TAGHAVI, S.A.; MARIÑO, M.A.; ROLSTON, D.E. Infiltration from trickle irrigation source. Journal of Irrigation and Drainage Engineering. New York, v.110, n.4, p.331-341, 1984. 